TRANSACTIONS OF THE

AMERICAN MATHEMATICAL SOCIETY

Volume 355, Number 3, Pages 1009-1024

S 0002-9947(02)03186-0

Article electronically published on November 1, 2002

\title{
CYCLIC COVERS OF RINGS WITH RATIONAL SINGULARITIES
}

\author{
ANURAG K. SINGH \\ Dedicated to Professor Phillip Griffith on the occasion of his sixtieth birthday
}

\begin{abstract}
We examine some recent work of Phillip Griffith on étale covers and fibered products from the point of view of tight closure theory. While it is known that cyclic covers of Gorenstein rings with rational singularities are Cohen-Macaulay, we show this is not true in general in the absence of the Gorenstein hypothesis. Specifically, we show that the canonical cover of a $\mathbb{Q}$-Gorenstein ring with rational singularities need not be Cohen-Macaulay.
\end{abstract}

\section{INTRODUCTION}

The theory of cyclic covers has found frequent use in commutative algebra in recent years. In $\mathrm{GrW}$ Griffith and Weston applied this theory in their study of torsion elements in the kernel of certain divisor class group homomorphisms. More recently, there has been a substantial amount of interest in cyclic covers arising from tight closure theory and its connections with the study of singularities in characteristic zero; see [Sm2], in particular, $\S 4$. While these connections have been illuminated and greatly advanced by studying the notions of F-rationality and Fregularity that arise from tight closure theory, the property of F-regularity remains something of a mystery. It is known that a Gorenstein ring is F-regular precisely if it is F-rational, and it is in the Gorenstein setting that the property of F-regularity is perhaps best understood. A frequent theme has been to extend results that are known for Gorenstein rings to the class of $\mathbb{Q}$-Gorenstein rings, and it is here that cyclic covers make their appearance. As an example, Hochster and Huneke showed that a Gorenstein integral domain of characteristic $p>0$ is a direct summand of every module-finite extension ring precisely if it is F-regular, [HH4]; the question arises whether this is true without the Gorenstein hypothesis, and the (albeit partial) answer to this now available uses cyclic covers, see [Si1]. In a geometric vein, Hara's theorem that a ring of characteristic zero with log terminal singularities has F-regular type uses a cyclic cover argument to reduce to the Gorenstein case where log terminal singularities agree with rational singularities, see [Ha]. It should be mentioned that rings of dimension two with rational singularities are $\mathbb{Q}$-Gorenstein

Received by the editors August 21, 2002.

2000 Mathematics Subject Classification. Primary 13A35, 13A02; Secondary 13H10, 14 B05.

This manuscript is based on work supported in part by the National Science Foundation under Grant No. DMS 0070268. I would like to thank the referee for a careful reading of the manuscript and for helpful suggestions. 
by the work of Lipman, Li], and so it is only natural to consider their cyclic canonical covers, which have the advantage of being Gorenstein rings.

In [Wa3] Watanabe showed that a cyclic cover of an F-regular ring is also Fregular and, as a consequence, that it is Cohen-Macaulay. One of the goals of this paper is to show that under the weaker hypothesis that a ring $R$ is F-rational (or that it has rational singularities), a cyclic cover of $R$ need not be a CohenMacaulay ring, even in the most natural setting where the cyclic cover is precisely the canonical cover of a $\mathbb{Q}$-Gorenstein ring $R$. Our main result is

Theorem 1.1. For all $d \geq 3$ there exists an $\mathbb{N}$-graded $\mathbb{Q}$-Gorenstein ring $R$ of dimension d, with an isolated rational singularity at its homogeneous maximal ideal, such that the canonical cover of $R$ is not a Cohen-Macaulay ring.

In section 5 we provide a tight closure approach to some results from Griffith's recent work $\mathrm{Gr}$. The question under consideration is the following. Let $f: X \rightarrow$ $Y$ be a finite étale morphism of smooth projective varieties over an algebraically closed field of characteristic zero. If $Y$ is arithmetically Cohen-Macaulay, does this property ascend to $X$ ? More precisely, if $\bigoplus_{n>0} H^{0}\left(Y, \mathcal{L}^{\otimes n}\right)$ is a Cohen-Macaulay homogeneous coordinate ring for $Y$, where $\mathcal{L}$ is an ample line bundle on $Y$, is the corresponding homogeneous coordinate ring $\bigoplus_{n \geq 0} H^{0}\left(X, f^{*} \mathcal{L}^{\otimes n}\right)$ of $X$ also Cohen-Macaulay? Similarly, one may ask the following question. Let $(R, m) \hookrightarrow$ $(S, n)$ be a module-finite extension of local or $\mathbb{N}$-graded rings which is étale on the punctured spectrum, Spec $R-\{m\}$. If $R$ is Cohen-Macaulay, is the ring $S$ also Cohen-Macaulay? The answer is negative, as established by examples of Griffith, and we show how a very general and interesting source of such examples comes from tight closure theory.

\section{GRAdED RINGS AND LOCAL COHOMOLOGY}

We shall follow the notation and conventions of [GoW]. For the convenience of the reader we record some standard facts about graded local cohomology modules and Segre products.

By an $\mathbb{N}$-graded ring $R$, we will always mean a ring $R=\bigoplus_{n>0} R_{n}$, finitely generated over a field $R_{0}=K$. We use $m_{R}$ or $m$ to denote the homogeneous maximal ideal $R_{+}$of $R$. By a homogeneous system of parameters for $R$ we mean a sequence of homogeneous elements of $R$ whose images form a system of parameters for $R_{m}$. In specific examples involving homomorphic images of polynomial rings, lowercase letters will denote the images of the corresponding variables, the variables being denoted by uppercase letters.

For a graded $R$-module $M$, the $i$ th graded component of $M$ will be denoted by $[M]_{i}$. We use $M(n)$ to denote the graded $R$-module with $[M(n)]_{i}=[M]_{n+i}$ for all $i \in \mathbb{Z}$. For graded $R$-modules $M$ and $N$, we may consider the graded $R$-module $\underline{\operatorname{Hom}}_{R}(M, N)$ whose $i$ th graded component $\left[\underline{\operatorname{Hom}}_{R}(M, N)\right]_{i}$ is the abelian group consisting of all graded (i.e., degree-preserving) $R$-linear homomorphisms from $M$ to $N(i)$. It is easy to verify that

$$
\underline{\operatorname{Hom}}_{R}(M, N(n))=\underline{\operatorname{Hom}}_{R}(M, N)(n)=\underline{\operatorname{Hom}}_{R}(M(-n), N) .
$$

Graded $R$-modules form an abelian category with enough injectives. For an integer $i \geq 0$, the functor $\underline{\operatorname{Ext}}_{R}^{i}(M,-)$ denotes the $i$ th derived functor of $\underline{\operatorname{Hom}}_{R}(M,-)$. If 
$M$ is a finitely generated graded $R$-module, then

$$
\underline{\operatorname{Hom}}_{R}(M, N)=\operatorname{Hom}_{R}(M, N) \text { and } \underline{\operatorname{Ext}}_{R}^{i}(M, N)=\operatorname{Ext}_{R}^{i}(M, N)
$$

as underlying $R$-modules, where $N$ is any graded $R$-module. For a homogeneous ideal $I$ of $R$ and a graded $R$-module $M$, the graded local cohomology modules of $M$ with support in $I$ (more precisely, with support in the closed set $V(I) \subseteq \operatorname{Spec} R$ ) are defined as

$$
\underline{H}_{I}^{i}(M)=\underline{\lim }_{n} \underline{\operatorname{Ext}}_{R}^{i}\left(R / I^{n}, M\right) .
$$

Since the modules $R / I^{n}$ are finitely generated, $\underline{H}_{I}^{i}(M)$ and $H_{I}^{i}(M)$ agree as underlying $R$-modules. If $\operatorname{dim} R=d$ and $x_{1}, \ldots, x_{d}$ is a homogeneous system of parameters for $R$, the module $\underline{H}_{m}^{d}(R)$ may be identified with

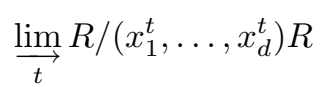

where the maps are induced by multiplication by the element $x_{1} \cdots x_{d} \in R$. Under the grading on $\underline{H}_{m}^{d}(R)$, we have

$$
\operatorname{deg}\left[z+\left(x_{1}^{t}, \ldots, x_{d}^{t}\right)\right]=\operatorname{deg} z-t \sum_{i=1}^{d} \operatorname{deg} x_{i}
$$

where $z$ is a homogeneous element of $R$. Goto and Watanabe defined $a(R)$, the $a$-invariant of $R$, as the highest integer $a$ such that $\left[\underline{H}_{m}^{d}(R)\right]_{a}$ is nonzero.

The injective hull of $K$ in the category of graded $R$-modules will be denoted by $\underline{E}_{R}(K)$, and this is also the injective hull of $K$ as an $R$-module. For a graded $R$-module $M$, its graded $K$-dual is $M^{*}=\underline{\operatorname{Hom}}_{K}(M, K)$ where

$$
\left[M^{*}\right]_{i}=\operatorname{Hom}_{K}(M, K(i))=\operatorname{Hom}_{K}(M(-i), K) .
$$

With this notation it turns out that $\underline{E}_{R}(K)=R^{*}$, and so

$$
\underline{\operatorname{Hom}}_{R}\left(M, \underline{E}_{R}(K)\right) \cong \underline{\operatorname{Hom}}_{K}(M, K)=M^{*} .
$$

The graded canonical module of $R$ is

$$
\omega_{R}=\left(\underline{H}_{m}^{d}(R)\right)^{*} \cong \underline{\operatorname{Hom}}_{R}\left(\underline{H}_{m}^{d}(R), \underline{E}_{R}(K)\right) .
$$

If the ring $R$ is Gorenstein, it is easy to see that there is a graded isomorphism $\omega_{R} \cong R(a)$ where $a$ is the $a$-invariant of the ring $R$.

Segre products. Let $A$ and $B$ be $\mathbb{N}$-graded rings over a field $A_{0}=B_{0}=K$. The Segre product of $A$ and $B$ is the ring

$$
R=A \# B=\bigoplus_{n \geq 0} A_{n} \otimes_{K} B_{n} .
$$

This has a natural grading in which $R_{n}=A_{n} \otimes_{K} B_{n}$. If $M$ and $N$ are graded modules over $A$ and $B$ respectively, then their Segre product is the graded $R$ module

$$
M \# N=\bigoplus_{n \in \mathbb{Z}}[M]_{n} \otimes_{K}[N]_{n} \quad \text { with } \quad[M \# N]_{n}=[M]_{n} \otimes_{K}[N]_{n} .
$$

We assume next that $A$ and $B$ are normal rings over an algebraically closed field $K$. In this case $R=A \# B$ is also a normal ring, since it is a direct summand of the 
normal ring $A \otimes_{K} B$. For reflexive modules $M$ and $N$ over $A$ and $B$ respectively, we have the Künneth formula for local cohomology, [GoW, Theorem 4.1.5]:

$$
\begin{aligned}
\underline{H}_{m_{R}}^{k}(M \# N) \cong & \left(M \# \underline{H}_{m_{B}}^{k}(N)\right) \oplus\left(\underline{H}_{m_{A}}^{k}(M) \# N\right) \\
& \oplus \bigoplus_{i+j=k+1}\left(\underline{H}_{m_{A}}^{i}(M) \# \underline{H}_{m_{B}}^{j}(N)\right) \text { for all } k \geq 0 .
\end{aligned}
$$

If $\operatorname{dim} A=r \geq 1$ and $\operatorname{dim} B=s \geq 1$, the above formula shows that $\operatorname{dim} R=r+s-1$. Furthermore, if $r \geq 2$ and $s \geq 2$, we get

$$
\underline{H}_{m_{R}}^{r+s-1}(R) \cong \underline{H}_{m_{A}}^{r}(A) \# \underline{H}_{m_{B}}^{s}(B),
$$

and, applying $\underline{\operatorname{Hom}}_{K}(-, K)$, this shows that $\omega_{R} \cong \omega_{A} \# \omega_{B}$.

In the paper $\mathrm{Ch}$, Chow established necessary and sufficient conditions under which the Segre product of two graded Cohen-Macaulay rings is Cohen-Macaulay. Let $U \hookrightarrow \mathbb{P}^{r}$ and $V \hookrightarrow \mathbb{P}^{s}$ be projective varieties with homogeneous coordinate rings $A$ and $B$ respectively. The Segre product $A \# B$ is a homogeneous coordinate ring for the Segre embedding $U \times V \hookrightarrow \mathbb{P}^{r s+r+s}$, and the question whether $U \times V \hookrightarrow \mathbb{P}^{r s+r+s}$ is projectively Cohen-Macaulay is precisely the question whether $A \# B$ is a CohenMacaulay ring. Chow's work was motivated by a question of Dwork and Ireland that arose from studying zeta functions of algebraic varieties. In [HR, \$14] Hochster and Roberts showed that under mild hypotheses on $A$ and $B$, Chow's results can be obtained by an application of the Künneth formula for sheaf cohomology. Also, Goto and Watanabe established such a result using the Künneth formula for local cohomology. Their result is

Theorem 2.1 ([GoW, Theorem 4.2.3]). Let $A$ and $B$ be $\mathbb{N}$-graded Cohen-Macaulay rings of dimension at least two. If $a(A)<0$ and $a(B)<0$, then the Segre product $A \# B$ is Cohen-Macaulay and $a(A \# B)<0$. Conversely if $A \# B$ is Cohen-Macaulay and the rings $A$ and $B$ contain nonzero homogeneous elements of all positive degrees, then $a(A)<0$ and $a(B)<0$.

Rational coefficient Weil divisors. We recall some notation and results from [De], Wa1], Wa3].

By a rational coefficient Weil divisor (or a $\mathbb{Q}$-divisor) on a normal projective variety $X$, we mean a $\mathbb{Q}$-linear combination of codimension-one irreducible subvarieties of $X$. If $D=\sum n_{i} V_{i}$, where $n_{i} \in \mathbb{Q}$ and $V_{i}$ are distinct irreducible subvarieties, we set $[D]=\sum\left[n_{i}\right] V_{i}$, where $[n]$ denotes the greatest integer less than or equal to $n$, and define $\mathcal{O}_{X}(D)=\mathcal{O}_{X}([D])$.

Let $D=\sum\left(p_{i} / q_{i}\right) V_{i}$ where the integers $p_{i}$ and $q_{i}$ are relatively prime, $q_{i}>0$, and the subvarieties $V_{i}$ are distinct. The fractional part of $D$ is defined to be the $\mathbb{Q}$-divisor $D^{\prime}=\sum\left(\left(q_{i}-1\right) / q_{i}\right) V_{i}$. The reason for this definition is that we then have the equality $-[-n D]=\left[n D+D^{\prime}\right]$ for any integer $n$, and this will be of use when we use Serre duality with $\mathbb{Q}$-divisors.

For an ample $\mathbb{Q}$-divisor $D$ (i.e., such that $n D$ is an ample Cartier divisor for some $n \in \mathbb{N}$ ), the generalized section ring corresponding to $D$ is

$$
R=R(X, D)=\bigoplus_{n \geq 0} H^{0}\left(X, \mathcal{O}_{X}(n D)\right) .
$$

With this notation, Demazure's result ([De, 3.5]) states that every $\mathbb{N}$-graded normal $\operatorname{ring} R$ is isomorphic to a generalized section ring $R(X, D)$ for some ample $\mathbb{Q}$-divisor $D$ on $X=\operatorname{Proj} R$. 
Let $X$ be a smooth projective variety of dimension $d$ with canonical divisor $K_{X}$, and let $D$ be an ample $\mathbb{Q}$-divisor on $X$. Let $\omega_{R}$ denotes the graded canonical module of the ring $R=R(X, D)$ and $\omega_{R}^{(i)}$ its $i$ th symbolic power. In the papers Wa1] and Wa3] Watanabe showed that

$$
\begin{aligned}
{\left[\underline{H}_{m}^{i}(R)\right]_{n} } & \cong H^{i-1}\left(X, \mathcal{O}_{X}(n D)\right) \quad \text { for all } \quad i \geq 2, \\
{\left[\omega_{R}^{(i)}\right]_{n} } & \cong H^{0}\left(X, \mathcal{O}_{X}\left(i\left(K_{X}+D^{\prime}\right)+n D\right)\right), \quad \text { and } \\
{\left[\underline{H}_{m}^{d+1}\left(\omega_{R}^{(i)}\right)\right]_{n} } & \cong H^{d}\left(X, \mathcal{O}_{X}\left(i\left(K_{X}+D^{\prime}\right)+n D\right)\right) .
\end{aligned}
$$

Rational singularities. We shall say that a normal ring $R$ essentially of finite type over a field of characteristic zero has rational singularities if the affine scheme Spec $R$ has only rational singularities. We recall a theorem of Watanabe:

Theorem 2.2 ([Wa2, Theorem 2.2]). Let $R$ be a normal $\mathbb{N}$-graded ring which is finitely generated over a field $R_{0}=K$ of characteristic zero. Then $R$ has rational singularities if and only if the following two conditions are satisfied:

(1) the open set Spec $R-\left\{m_{R}\right\}$ has only rational singularities, and

(2) the ring $R$ is Cohen-Macaulay with $a(R)<0$.

Note that condition (1) above is satisfied if the ring $R$ has an isolated singularity at its homogeneous maximal ideal $m_{R}$. An application of this theorem, which we will have use for later, is the following:

Proposition 2.3. Let $D$ be an effective $\mathbb{Q}$-divisor on projective space $\mathbb{P}^{d}$ over a field of characteristic zero, and consider the generalized section ring $R=R\left(\mathbb{P}^{d}, D\right)$. If Spec $R-\left\{m_{R}\right\}$ has at most rational singularities, then $R$ has rational singularities.

Proof. By construction $R$ is a normal ring of dimension $d+1$, and since

$$
\left[\underline{H}_{m}^{i}(R)\right]_{n} \cong H^{i-1}\left(\mathbb{P}^{d}, \mathcal{O}_{\mathbb{P}^{d}}(n D)\right)=0
$$

for all $2 \leq i \leq d$, for all $n \in \mathbb{Z}$, the ring $R$ must be Cohen-Macaulay. It remains only to verify that $a(R)<0$. Using * to denote the Serre dual,

$$
\left[\underline{H}_{m}^{d+1}(R)\right]_{n} \cong H^{d}\left(\mathbb{P}^{d}, \mathcal{O}_{\mathbb{P}^{d}}(n D)\right) \cong H^{0}\left(\mathbb{P}^{d}, \mathcal{O}_{\mathbb{P}^{d}}\left(K_{\mathbb{P}^{d}}+D^{\prime}-n D\right)\right)^{*},
$$

which is zero for all $n \geq 0$ since $D$ is effective.

\section{Tight Closure}

For the theory of tight closure, developed by Hochster and Huneke, we refer the reader to the papers [HH1], $\mathrm{HH} 2, \mathrm{HH} 3$ and $\mathrm{HH} 4$. In our applications of this theory, we will usually be restricting ourselves to the class of $\mathbb{N}$-graded rings, and we summarize some results which will suffice for our needs. The reader can find more general forms of several of these results in the papers mentioned above.

Theorem 3.1. Let $R$ be an $\mathbb{N}$-graded ring over a perfect field $R_{0}=K$ of characteristic $p>0$.

(1) If $R$ is a regular ring, then it is strongly F-regular.

(2) Direct summands of F-regular rings are F-regular.

(3) If $R$ is an F-rational ring, then it is normal and Cohen-Macaulay.

(4) An F-rational Gorenstein ring is F-regular.

(5) The ring $R$ is F-regular if and only if it is strongly F-regular. 
Proof. For (1) - (4) see [HH1, Theorem 3.1] and [HH3, Theorem 4.2], and for (5), see [LS, Corollary 4.3].

In [HH5] Hochster and Huneke have developed a theory of tight closure for rings essentially of finite type over fields of characteristic zero. However, notions corresponding to F-regularity and F-rationality can also be defined in characteristic zero without explicitly considering a closure operation for rings of characteristic zero. A ring $R=K\left[X_{1}, \ldots, X_{n}\right] / I$ over a field $K$ of characteristic zero is said to be of dense $F$-regular type if there exists a finitely generated $\mathbb{Z}$-algebra $A \subseteq K$ and a finitely generated free $A$-algebra

$$
R_{A}=A\left[X_{1}, \ldots, X_{n}\right] / I_{A}
$$

such that $R \cong R_{A} \otimes_{A} K$ and, for all maximal ideals $\mu$ in a Zariski dense subset of Spec $A$, the fiber rings $R_{A} \otimes_{A} A / \mu$ are F-regular rings of characteristic $p>0$. Similarly, $R$ is of dense F-rational type if for a dense subset of $\mu$, the fiber rings $R_{A} \otimes_{A} A / \mu$ are F-rational. Combining some of the results from [Ha], [HW], SSm1, we have

Theorem 3.2 (Hara-Smith-Watanabe). Let $R$ be a ring finitely generated over a field of characteristic 0 . Then $R$ has rational singularities if and only if it is of dense F-rational type. If $R$ is $\mathbb{Q}$-Gorenstein, then it has log terminal singularities if and only if it is of dense F-regular type.

When used along with Proposition 2.3, the following result provides various examples of F-rational rings which are not F-regular:

Proposition 3.3. Let $D$ be an ample $\mathbb{Q}$-divisor on $\mathbb{P}^{d}$, where $\mathbb{P}^{d}$ is projective space over a perfect field $K$ of characteristic $p>0$ (or of characteristic 0 ), and consider the generalized section ring $R=R\left(\mathbb{P}^{d}, D\right)$. If $R$ is F-regular (respectively, of dense F-regular type $)$, then $\operatorname{deg}\left(K_{\mathbb{P}^{d}}+D^{\prime}\right)<0$.

Proof. If $K$ is a perfect field of characteristic $p>0$, the $\operatorname{ring} R$ is F-finite, i.e., the $\operatorname{ring} R^{1 / p}$ (obtained by adjoining $p$ th roots of elements of $R$ ) is a finitely generated $R$-module. By [LS], $R$ is strongly F-regular and $0_{E}^{*}=0$, where $E$ is the injective hull of the residue field $K$; in other words, the zero submodule of $E$ is tightly closed. Let $\zeta \in\left[H_{m}^{d+1}\left(\omega_{R}\right)\right]_{0}$ denote a socle generator of $E$. Since $0_{E}^{*}=0$, for any nonzero element $c \in R_{n}$, there exists $q=p^{e}$ such that the element

$$
c F^{e}(\zeta) \in\left[H_{m}^{d+1}\left(\omega_{R}^{(q)}\right)\right]_{n}
$$

is nonzero, where $F$ denotes the Frobenius morphism. Consequently

$$
\left[H_{m}^{d+1}\left(\omega_{R}^{(q)}\right)\right]_{n} \cong H^{d}\left(\mathbb{P}^{d}, \mathcal{O}_{\mathbb{P}^{d}}\left(q\left(K_{\mathbb{P}^{d}}+D^{\prime}\right)+n D\right)\right) \neq 0,
$$

and it follows that $\operatorname{deg}\left(K_{\mathbb{P} d}+D^{\prime}\right)<0$.

Example 3.4. For an algebraically closed field $K$ of characteristic 0 and positive integers $r, n$, and $m \geq 3$, consider the $\mathbb{Q}$-divisor

$$
D=\frac{1}{r} V\left(X_{1}^{n}+\cdots+X_{m}^{n}\right) \quad \text { on } \quad \mathbb{P}^{m-1}=\operatorname{Proj} K\left[X_{1}, \ldots, X_{m}\right] .
$$

Note that $X_{1}^{n}+\cdots+X_{m}^{n}$ is irreducible, since $m \geq 3$. Take the corresponding generalized section ring $R=\bigoplus_{n \geq 0} H^{0}\left(\mathbb{P}^{m-1}, \mathcal{O}_{\mathbb{P}^{m-1}}(n D)\right) Z^{n}$, where the term $Z^{n}$ 
serves to keep track of the degree of an element of $R$. The ring $R$ is generated over the field $K$ by $Z$, and elements of the form

$$
\frac{\mu_{n}\left(X_{1}, \ldots, X_{m}\right)}{X_{1}^{n}+\cdots+X_{m}^{n}} Z^{r}
$$

where $\mu_{n}\left(X_{1}, \ldots, X_{m}\right)$ is a monomial of degree $n$ in the variables $X_{1}, \ldots, X_{m}$. Consequently $R$ may be identified with the subring of the hypersurface

$$
S=K\left[Z, X_{1}, \ldots, X_{m}\right] /\left(Z^{r}-\left(X_{1}^{n}+\cdots+X_{m}^{n}\right)\right)
$$

generated by $z$ (i.e., the image of $Z$ in $S$ ) and monomials of degree $n$ in $x_{1}, \ldots, x_{m}$ (the images, respectively, of $X_{1}, \ldots, X_{m}$ ). For another description of $R$, let $\zeta \in K$ be a primitive $n$th root of unity and consider the $K$-automorphism $\sigma$ of $S$ where $\sigma\left(x_{i}\right)=\zeta x_{i}$ for $1 \leq i \leq m$ and $\sigma(z)=z$. If $G=(\sigma) \cong \mathbb{Z} / n \mathbb{Z}$, then the ring of invariants of this group action, $S^{G}$, is isomorphic to $R$. It is easy to verify that $R$ has an isolated singularity at its homogeneous maximal ideal $m_{R}$, since, for all $1 \leq i \leq m$, the localization $R_{x_{i}^{n}}$ is a regular ring. The $\mathbb{Q}$-divisor $D$ is effective, and so the ring $R$ has rational singularities by Proposition 2.3. If $R$ is of dense F-regular type, then, by Proposition 3.3 ,

$$
\operatorname{deg}\left(K_{\mathbb{P} m-1}+D^{\prime}\right)=-m+\left(\frac{r-1}{r}\right) n<0 .
$$

Consequently if $(r-1) n-m r \geq 0$, then $R$ is a $\mathbb{Q}$-Gorenstein ring, with an isolated rational singularity, which is not of dense F-regular type.

\section{Cyclic covers}

For the general theory of cyclic covers, we refer the reader to the paper of Tomari and Watanabe, [TW]. The discussion below shall suffice for our needs.

Let $R$ be an $\mathbb{N}$-graded or local normal domain with an ideal $I$ of pure height one (i.e., a divisorial ideal) which has finite order $n$ when regarded as an element of the divisor class group of the ring $R$. Let $I^{(n)}=u R$ where, in the graded case, we assume furthermore that $I$ and $u$ are homogeneous. By the cyclic cover of $R$ with respect to $I$, we mean the ring

$$
S=R\left[I t, I^{(2)} t^{2}, \ldots, I^{(i)} t^{i}, \ldots\right] /\left(u t^{n}-1\right) .
$$

This ring is finitely generated as an $R$-module; in particular, the generators of $I t, \ldots, I^{(n-1)} t^{n-1}$ form a generating set for $S$. If the characteristic of the residue field of $R$ is relatively prime to $n$, the inclusion of $R$ in $S$ is étale in codimension one; more generally, if $P \in \mathrm{Spec} R$ is such that $I R_{P}$ is principal, say $I R_{P}=v R_{P}$, then $u=\lambda v^{n}$ for a unit $\lambda \in R_{P}$, and so

$$
S_{P}=R_{P}[v t] /\left(\lambda(v t)^{n}-1\right),
$$

which is an étale extension of $R_{P}$. Note that $S$ is a normal domain. We next recall a theorem of Watanabe:

Theorem 4.1 ([Wa3, Theorem 2.7]). Let $R \rightarrow S$ be a finite local homomorphism of normal local rings (or a graded homomorphism of $\mathbb{N}$-graded rings) which is étale in codimension one. If the ring $R$ is strongly F-regular, then the ring $S$ is also strongly F-regular. In particular, if $S$ is the cyclic cover of a strongly F-regular ring $R$ with respect to a divisorial ideal of order $n$, then $S$ is strongly F-regular (and hence Cohen-Macaulay) provided the characteristic of $R$ does not divide $n$. 
One of the main goals of this paper is to show that under the weaker hypothesis that the ring $R$ is F-rational, a cyclic cover $S$ need not be Cohen-Macaulay even if $R$ is a $\mathbb{Q}$-Gorenstein normal domain with an isolated rational singularity, and the finite extension $S$ is simply the canonical cover of the ring $R$.

We focus next on the case where $R$ is an $\mathbb{N}$-graded normal domain, and $I$ is a homogeneous ideal of pure height one. In this setting we show that there is a natural $\mathbb{Q}$-grading on the cyclic cover $S$ which extends the grading on $R$. The main point of our result below is that this grading involves only nonnegative rational numbers, and that the degree zero component of $S$ is the field $[R]_{0}=K$.

Proposition 4.2. Let $R$ be an $\mathbb{N}$-graded normal domain where $[R]_{0}=K$ is a field, and let $I$ be a homogeneous ideal of pure height one which has order $n$ as an element of the divisor class group of $R$. Then $I^{(n)}=u R$ for a homogeneous element $u \in R$, and there is a unique $\mathbb{Q}$-grading on the cyclic cover

$$
S=R\left[I t, I^{(2)} t^{2}, \ldots, I^{(i)} t^{i}, \ldots\right] /\left(u t^{n}-1\right)
$$

which extends the grading on $R$. Under this grading, homogeneous elements of $S$ have nonnegative weights and $[S]_{0}=[R]_{0}=K$.

Proof. Since $u t^{n}=1$, we have $\operatorname{deg} t=-(\operatorname{deg} u) / n$ in the fraction field of $S$, and this gives the unique extension of the $R$-grading. Let $x t^{i} \in I^{(i)} t^{i}$ be a nonzero homogeneous element of $S$ where $1 \leq i \leq n-1$. Then

$$
\left(x t^{i}\right)^{n} \in I^{(n i)} t^{n i}=u^{i} t^{n i} R=R,
$$

and so $\operatorname{deg}\left(x t^{i}\right) \geq 0$. If $\operatorname{deg}\left(x t^{i}\right)=0$, then $(*)$ furthermore shows that $\left(x t^{i}\right)^{n}=c \in$ $[R]_{0}=K$, and so $x^{n}=c u^{i}$. Since $x \in I^{(i)}$, we have

$$
c u^{i}=x x^{n-1} \in I^{(i)} I^{(i n-i)} \subseteq I^{(i n)}=u^{i} R,
$$

and so $I^{(i)} I^{(i n-i)}=u^{i} R$. However, this means that $I^{(i)}$ is an invertible fractional ideal, and hence a projective $R$-module, $\mathrm{Ma}$, Theorem 11.3]. Since $R$ and $I^{(i)}$ are graded, $I^{(i)}$ must be a free $R$-module, and since it has rank one, it must be principal. However, this contradicts the fact that the order of $I$ in the divisor class group of $R$ is $n$.

We usually prefer to work with this $\mathbb{Q}$-grading, since it agrees with the grading on $R$. However, we point out that since only finitely many denominators occur in this $\mathbb{Q}$-grading, one could multiply by a suitable integer to obtain an $\mathbb{N}$-grading on $S$. (Specifically, multiplying all weights by the integer $n$ will ensure an $\mathbb{N}$-grading.)

Our focus next is on the canonical cover of a graded $\mathbb{Q}$-Gorenstein ring $R$. It is well known that if an integral domain $R$ has a canonical module, then there is an ideal of $R$ which is isomorphic to that canonical module $\omega$. However, in the graded situation it is not always possible to find an ideal of $R$ which is isomorphic to the graded canonical module $\underline{\operatorname{Hom}}_{K}\left(\underline{H}_{m}^{d}(R), K\right)$ in a degree-preserving manner. This obstacle may be circumvented by choosing instead a fractional ideal of $R$, and henceforth, by a graded canonical module, we shall mean a fractional ideal $\omega$ of $R$ which is isomorphic to $\underline{\operatorname{Hom}}_{K}\left(\underline{H}_{m}^{d}(R), K\right)$ via a degree-preserving isomorphism. Since $\omega$ is a reflexive module of rank one, its symbolic power $\omega^{(i)}$ may be defined as the double dual of the tensor product of $i$ copies of $\omega$.

An $\mathbb{N}$-graded or local normal domain $R$ is said to be $\mathbb{Q}$-Gorenstein if its canonical module has finite order when regarded as an element of the divisor class group of $R$. A generalized section ring $R\left(\mathbb{P}^{d}, D\right)$ is always $\mathbb{Q}$-Gorenstein: since $\operatorname{Pic}\left(\mathbb{P}^{d}\right)=\mathbb{Z}$, 
there exist integers $m$ and $n$ such that $m\left(K_{\mathbb{P}^{d}}+D^{\prime}\right)$ and $n D$ are linearly equivalent Weil divisors. By the canonical cover of a $\mathbb{Q}$-Gorenstein ring $R$, we mean the cyclic cover of $R$ with respect to its canonical module. For a $\mathbb{Q}$-Gorenstein ring $R$, we shall use $\widetilde{R}$ to denote its canonical cover.

We shall say that a normal $\mathbb{N}$-graded ring $R$ is quasi-Gorenstein if the module $\underline{\operatorname{Hom}}_{K}\left(\underline{H}_{m}^{d}(R), K\right)$ and the ring $R$ are isomorphic as underlying $R$-modules. In this case, it follows that

$$
\omega_{R}=\left(\underline{H}_{m}^{d}(R)\right)^{*} \cong R(a),
$$

where $a$ is the $a$-invariant of the ring $R$. A quasi-Gorenstein Cohen-Macaulay ring is Gorenstein.

Proposition 4.3. Let $R$ be a graded normal $\mathbb{Q}$-Gorenstein ring. If the canonical module $\omega$ of $R$ has order $n$ in the divisor class group of $R$ and $\omega^{(n)}=u R$, consider the graded canonical cover

$$
\widetilde{R}=R\left[\omega t, \omega^{(2)} t^{2}, \ldots, \omega^{(n-1)} t^{n-1}, \ldots\right] /\left(u t^{n}-1\right)
$$

as above, i.e., with $\operatorname{deg} t=-(\operatorname{deg} u) / n$. Then the ring $\widetilde{R}$ is quasi-Gorenstein with $a$-invariant $a(\widetilde{R})=-(\operatorname{deg} u) / n$.

Proof. If $k=(\operatorname{deg} u) / n$, we have a graded isomorphism

$$
\widetilde{R} \cong R \oplus \omega(k) \oplus \omega^{(2)}(2 k) \oplus \cdots \oplus \omega^{(n-1)}(n k-k) .
$$

If $d=\operatorname{dim} R$ and $0 \leq i \leq n-1$, there are graded isomorphisms

$$
\begin{aligned}
\underline{\operatorname{Hom}}_{K}\left(\underline{H}_{m}^{d}\left(\omega^{(i)}\right), K\right) & \cong \underline{\operatorname{Hom}}_{K}\left(\underline{H}_{m}^{d}(\omega) \underline{\otimes}_{R} \omega^{(i-1)}, K\right) \\
& \cong \underline{\operatorname{Hom}}_{R}\left(\omega^{(i-1)}, \underline{\operatorname{Hom}}_{K}\left(\underline{H}_{m}^{d}(\omega), K\right)\right) \\
& \cong \underline{\operatorname{Hom}}_{R}\left(\omega^{(i-1)}, R\right) \cong \omega^{(1-i)} .
\end{aligned}
$$

Consequently

$$
\begin{aligned}
\omega_{\widetilde{R}} & \cong \underline{\operatorname{Hom}}_{K}\left(\underline{H}_{m}^{d}(\widetilde{R}), K\right) \cong \bigoplus_{i=0}^{n-1} \underline{\operatorname{Hom}}\left(\underline{H}_{m}^{d}\left(\omega^{(i)}\right)(i k), K\right) \\
& \cong \bigoplus_{i=0}^{n-1} \omega^{(1-i)}(-i k) \cong \widetilde{R}(-k),
\end{aligned}
$$

and so $\widetilde{R}$ is quasi-Gorenstein with $a$-invariant $a(\widetilde{R})=-k=-(\operatorname{deg} u) / n$.

We record an example that we shall use later:

Example 4.4. Let $K$ be an algebraically closed field of characteristic 0, and take the $\mathbb{Q}$-divisor

$$
D=\frac{1}{3} V\left(y_{0}\right)+\frac{1}{3} V\left(z_{0}\right)+\frac{1}{3} V\left(y_{0}+z_{0}\right) \quad \text { on } \quad \mathbb{P}^{1}=\operatorname{Proj} K\left[y_{0}, z_{0}\right]
$$

where, for example, $V\left(y_{0}\right)$ denotes the point of $\mathbb{P}^{1}$ defined by the vanishing of $y_{0}$. Setting $A=\bigoplus_{n \geq 0} H^{0}\left(\mathbb{P}^{1}, \mathcal{O}_{\mathbb{P}^{1}}(n D)\right) x^{n}$, the generators of $A$ are

$$
\frac{y_{0}^{3} x^{3}}{y_{0} z_{0}\left(y_{0}+z_{0}\right)}, \frac{y_{0}^{2} z_{0} x^{3}}{y_{0} z_{0}\left(y_{0}+z_{0}\right)}, \frac{y_{0} z_{0}^{2} x^{3}}{y_{0} z_{0}\left(y_{0}+z_{0}\right)}, \frac{z_{0}^{3} x^{3}}{y_{0} z_{0}\left(y_{0}+z_{0}\right)}, \quad \text { and } \quad x,
$$

and so we may identify $A$ with the subring of the hypersurface

$$
H=K[X, Y, Z] /\left(X^{3}-Y Z(Y+Z)\right)
$$


generated by the elements $y^{3}, y^{2} z, y z^{2}, z^{3}$ and $x$. A graded canonical module for $A$ is

$$
\omega_{A}=\frac{1}{x^{2}}\left(y^{3}, y^{2} z\right) A
$$

and its symbolic powers are

$$
\omega_{A}^{(2)}=\frac{1}{x^{4}}\left(y^{6}, y^{5} z, y^{4} z^{2}\right) A \quad \text { and } \quad \omega_{A}^{(3)}=\frac{1}{x^{6}}\left(y^{6}\right) A .
$$

The isomorphism defined by

$$
\frac{y^{3} t}{x^{2}} \mapsto y, \frac{y^{2} z t}{x^{2}} \mapsto z, \frac{y^{6} t^{3}}{x^{6}} \mapsto 1
$$

may be used to identify $\widetilde{A}$ with the hypersurface $H$.

We next examine when the Segre product of two graded $\mathbb{Q}$-Gorenstein rings is a $\mathbb{Q}$-Gorenstein ring.

Proposition 4.5. Let $A$ and $B$ be normal $\mathbb{N}$-graded $\mathbb{Q}$-Gorenstein rings over an algebraically closed field $[A]_{0}=[B]_{0}=K$. If the graded canonical modules $\omega_{A}$ and $\omega_{B}$ have orders $m$ and $n$ respectively as divisor class group elements, choose homogeneous elements $u$ and $v$ in the fraction fields of $A$ and $B$ such that $\omega_{A}^{(m)} \cong u A$ and $\omega_{B}^{(n)} \cong v B$ as graded fractional ideals. If $n \operatorname{deg} u=m \operatorname{deg} v$, then the Segre product $R=A \# B$ is also a $\mathbb{Q}$-Gorenstein ring. If, furthermore, $m$ and $n$ are relatively prime integers, then the Segre product of the graded canonical covers of $A$ and $B$ is isomorphic to the canonical cover of $R=A \# B$, i.e.,

$$
\widetilde{A \# B} \cong \widetilde{A} \# \widetilde{B} \text {. }
$$

Proof. It is proved in GoW that the canonical module $\omega_{R}$ is the Segre product of the graded canonical modules, $\omega_{A} \# \omega_{B}$, see also $\S 2$. We have

$$
\omega_{R}^{(m n)} \cong \omega_{A}^{(m n)} \# \omega_{B}^{(m n)} \cong u^{n} A \# v^{m} B,
$$

and this is isomorphic to $u^{n} v^{m}(A \# B)$ whenever $n \operatorname{deg} u=m \operatorname{deg} v$.

In the case that $m$ and $n$ are relatively prime, $\omega_{R}$ has order $m n$ as a divisor class group element. If

$$
k=(\operatorname{deg} u) / m=(\operatorname{deg} v) / n,
$$

then the graded canonical cover of $R$ is

$$
\widetilde{R} \cong \bigoplus_{r=0}^{m n-1} \omega_{R}^{(r)}(k r) \cong \bigoplus_{r=0}^{m n-1} \omega_{A}^{(r)}(k r) \# \omega_{B}^{(r)}(k r) .
$$

Since $m$ and $n$ are relatively prime integers, given arbitrary $i, j \in \mathbb{N}$, there exists a unique integer $r$ with $0 \leq r \leq m n-1$ such that $r \equiv i \bmod m$ and $r \equiv j \bmod n$. Consequently

$$
\widetilde{R} \cong \bigoplus_{i=0}^{m-1} \bigoplus_{j=0}^{n-1} \omega_{A}^{(i)}(k i) \# \omega_{B}^{(j)}(k j) \cong \widetilde{A} \# \widetilde{B}
$$


By Watanabe's result, Theorem 4.1, the canonical cover of a $\mathbb{Q}$-Gorenstein strongly F-regular ring $R$ is also strongly F-regular, provided the characteristic of $R$ does not divide the order of $\left[\omega_{R}\right] \in \mathrm{Cl}(R)$. The corresponding result for log terminal singularities was proved earlier by Kawamata:

Theorem 4.6 ([Ka, Proposition 1.7]). Let $R$ be a normal $\mathbb{Q}$-Gorenstein ring of characteristic zero with a canonical cover $\widetilde{R}$. Then $R$ has log terminal singularities if and only if $\widetilde{R}$ has log terminal singularities.

Infinite covers. When the canonical ideal $\omega_{R}$ of a Cohen-Macaulay normal ring $(R, m)$ is not necessarily of finite order in the divisor class group $\mathrm{Cl}(R)$, the anticanonical cover $S$ may be constructed by taking an ideal $I$ of pure height one which is an inverse for $\omega_{R}$ in $\mathrm{Cl}(R)$, and forming the symbolic Rees ring

$$
S=\bigoplus_{i \geq 0} I^{(i)}
$$

The interest in the anti-canonical cover arises from the fact that if $S$ as above is Noetherian and Cohen-Macaulay, then it is Gorenstein. Symbolic Rees rings in general need not be Noetherian, but there is an interesting theorem of Watanabe that applies when the symbolic Rees ring is Noetherian:

Theorem 4.7 (Wa4 Theorem 0.1]). Let $(R, m)$ be a strongly F-regular ring, and $I$ an ideal of pure height one which is the inverse of the canonical module $\omega_{R}$ in $\mathrm{Cl}(R)$. If the anti-canonical cover $S=\bigoplus_{i \geq 0} I^{(i)}$ is Noetherian, then it is strongly F-regular.

For an arbitrary divisorial ideal $I$ of a strongly F-regular $\operatorname{ring} R$, Watanabe raised the question whether the symbolic Rees algebra $\mathcal{R}_{s}(I)=\bigoplus_{n \geq 0} I^{(n)}$ is CohenMacaulay whenever it is Noetherian. In Si2 we developed the notion of multisymbolic Rees algebras and used it to show that $\mathcal{R}_{s}(I)$ is indeed Cohen-Macaulay whenever a certain auxiliary ring is finitely generated over $R$. More precisely, we established:

Theorem 4.8 ([Si2, Theorem 5.1]). Let $(R, m)$ be a strongly F-regular ring with canonical ideal $\omega_{R}$. Given a divisorial ideal $I$, choose a divisorial ideal $J$ such that $[I]+[J]+\left[\omega_{R}\right]=0$ in the divisor class group $\mathrm{Cl}(R)$. If the multi-symbolic Rees algebra

$$
\mathcal{R}_{s}(I, J)=\bigoplus_{n, m \geq 0} \operatorname{Hom}_{R}\left(\operatorname{Hom}_{R}\left(I^{n} J^{m}, R\right), R\right)
$$

is finitely generated over $R$, then $\mathcal{R}_{s}(I)$ is strongly F-regular and, in particular, is Cohen-Macaulay.

Note that $\operatorname{Hom}_{R}\left(\operatorname{Hom}_{R}\left(I^{n} J^{m}, R\right), R\right)$ above is simply the reflexive hull of $I^{n} J^{m}$. The hypothesis that $R$ is strongly F-regular is indeed used in an essential way: Watanabe has constructed an example of an F-rational ring $R$ with a divisorial ideal $I$ such that the symbolic Rees algebra $\mathcal{R}_{s}(I)$ is not Cohen-Macaulay [Wa4, Example 4.4]. Our Theorem 6.1 may be viewed as a strengthening of this in the sense that we obtain such an example with the additional restriction that the divisorial ideal $I$ has finite order in $\mathrm{Cl}(R)$. 


\section{GRIFFIth's EXAMPLES FROM A TIGHT CLOSURE VIEWPOINT}

Let $f: X \rightarrow Y$ be a finite morphism of smooth projective varieties over an algebraically closed field $K$ of characteristic zero, and let $\mathcal{L}$ be an ample line bundle on $Y$ such that the homogeneous coordinate ring $\bigoplus_{n \geq 0} H^{0}\left(Y, \mathcal{L}^{\otimes n}\right)$ is Cohen-Macaulay. In $\mathrm{Gr}$ Griffith examined the issue of whether the arithmetically Cohen-Macaulay property ascended to $X$, i.e., whether $\bigoplus_{n \geq 0} H^{0}\left(X, f^{*} \mathcal{L}^{\otimes n}\right)$ is Cohen-Macaulay, under the hypothesis that the homomorphism

$$
\bigoplus_{n \geq 0} H^{0}\left(Y, \mathcal{L}^{\otimes n}\right) \rightarrow \bigoplus_{n \geq 0} H^{0}\left(X, f^{*} \mathcal{L}^{\otimes n}\right)
$$

is étale away from the irrelevant ideal. Griffith constructed examples of morphisms $f: X \rightarrow Y$ where the arithmetically Cohen-Macaulay property ascends but, if $r \geq 1$, it does not ascend for the induced morphism $f: X \times \mathbb{P}^{r} \rightarrow Y \times \mathbb{P}^{r}$.

We recall the examples from $[\mathrm{Gr}]$, and discuss them from the point of view of $\log$ terminal singularities. For $d \geq 4$, let $S$ be the hypersurface

$$
S=K\left[Z, X_{1}, \ldots, X_{d-1}\right] /\left(Z^{d}-\left(X_{1}^{d}+\cdots+X_{d-1}^{d}\right)\right)
$$

over an algebraically closed field $K$ of characteristic zero. Let $\zeta$ be a primitive $d$ th root of unity and consider the $K$-automorphism $\sigma$ of $S$ where $\sigma\left(x_{i}\right)=\zeta x_{i}$ for $1 \leq i \leq d-1$ and $\sigma(z)=z$. Let $G=(\sigma) \cong \mathbb{Z} / d \mathbb{Z}$. Then the ring of invariants of this group action $R=S^{G}$ is generated over $K$ by $z$ and the monomials of degree $d$ in the elements $x_{1}, \ldots, x_{d-1}$. Let $A=K\left[Y_{0}, \ldots, Y_{r}\right]$ be a polynomial ring over $K$ where $r \geq 1$, and set $X=\operatorname{Proj} S, Y=\operatorname{Proj} R$, and $\mathbb{P}^{r}=\operatorname{Proj} A$. In his paper, Griffith established the following results:

Proposition 5.1 ([Gr], $\S 4)$. With the above notation,

(1) The rings $R$ and $S$ are Cohen-Macaulay and the extension $R \rightarrow S$ is generically Galois, with Galois group $\mathbb{Z} / d \mathbb{Z}$. The extension of Segre products $R \# A$ $\rightarrow S \# A$ is also generically Galois, with Galois group $\mathbb{Z} / d \mathbb{Z}$.

(2) The graded $K$-algebra $R$ satisfies $a(R)<0$ whereas $a(S)=0$. Consequently the ring $R \# A$ is Cohen-Macaulay, but $S \# A$ is not. More specifically, $S \# A$ satisfies the Serre condition $S_{d-1}$ but not the condition $S_{d}$.

(3) The extension $R \rightarrow S$ is étale away from the irrelevant maximal ideal, as is the extension $R \# A \rightarrow S \# A$.

Remark 5.2. The ring $R$ above is the case of Example 3.4 with $r=n=d$ and $m=d-1$, i.e., it is isomorphic to the generalized section ring of the $\mathbb{Q}$-divisor

$$
D=\frac{1}{d} V\left(X_{1}^{d}+\cdots+X_{d-1}^{d}\right) \quad \text { on } \quad \operatorname{Proj} K\left[X_{1}, \ldots, X_{d-1}\right] .
$$

As a graded canonical module for $R$, we may take

$$
\omega_{R}=\frac{1}{z^{d-1}}\left(x_{1}^{d}, x_{1}^{d-1} x_{2}, \ldots, x_{1}^{d-1} x_{d-1}\right) R .
$$

This has order $d$ as an element of $\mathrm{Cl}(R)$, and $\omega_{R}^{(d)}=\left(x_{1}^{d(d-1)} / z^{d(d-1)}\right) R$. Furthermore, it turns out that the canonical cover of $R$ is isomorphic to the hypersurface $S$. Note that $\operatorname{deg}\left(K_{\mathbb{P}^{d-2}}+D^{\prime}\right)=0$, and so $R$ is an example of a $\mathbb{Q}$-Gorenstein ring with an isolated rational singularity which is not a log terminal singularity. Such a ring always yields an example in which the arithmetically Cohen-Macaulay property fails to ascend, as we record in the proposition below: 
Proposition 5.3. Let $R$ be an $\mathbb{N}$-graded $\mathbb{Q}$-Gorenstein ring of characteristic zero with an isolated rational singularity which is not a log terminal singularity. Then at least one of the following two conditions holds:

(1) The canonical cover $\widetilde{R}$ of the ring $R$ is not Cohen-Macaulay-in other words, the arithmetically Cohen-Macaulay property does not ascend under the morphism Proj $\widetilde{R} \rightarrow \operatorname{Proj} R$.

(2) The canonical cover satisfies $a(\widetilde{R}) \geq 0$, and so, if $r \geq 1$, the arithmetically Cohen-Macaulay property does not ascend under the morphism

$$
(\operatorname{Proj} \widetilde{R}) \times \mathbb{P}^{r} \rightarrow(\operatorname{Proj} R) \times \mathbb{P}^{r} .
$$

Proof. By Kawamata's result, recorded earlier as Theorem 4.6 the canonical cover $\widetilde{R}$ does not have log terminal singularities. But then, since $\widetilde{R}$ is quasi-Gorenstein, it cannot have rational singularities. By our assumption $R$ has an isolated singularity, and therefore the canonical cover $\widetilde{R}$ has an isolated singularity as well. By Watanabe's result, Theorem 2.2, the failure of $\widetilde{R}$ to have rational singularities can be attributed to one of the following two reasons:

(1) The ring $\widetilde{R}$ is not Cohen-Macaulay, and so the arithmetically CohenMacaulay property does not ascend under the morphism Proj $\widetilde{R} \rightarrow \operatorname{Proj} R$.

(2) The ring $\widetilde{R}$ has a nonnegative $a$-invariant. In this case, consider the polynomial ring $A=K\left[Y_{0}, \ldots, Y_{r}\right]$ where $r \geq 1$. Then $R \# A$ is a Cohen-Macaulay ring whereas, by the Künneth formula for local cohomology, $\widetilde{R} \# A$ is not CohenMacaulay. Hence the arithmetically Cohen-Macaulay property does not ascend under the morphism $(\operatorname{Proj} \widetilde{R}) \times \mathbb{P}^{r} \rightarrow(\operatorname{Proj} R) \times \mathbb{P}^{r}$ for $r \geq 1$.

The canonical cover $\widetilde{R}$ of the ring $R$ constructed by Griffith is isomorphic to the hypersurface

$$
S=K\left[X_{1}, \ldots, X_{d-1}, Z\right] /\left(Z^{d}-\left(X_{1}^{d}+\cdots+X_{d-1}^{d}\right)\right),
$$

which, while being Cohen-Macaulay, does not have rational singularities since $a(S)=0$, i.e., due to reason (2) above. The question then arises: does there exist a ring $R$, with an isolated rational singularity, whose canonical cover $\widetilde{R}$ does not have rational singularities for reason (1), i.e., because $\widetilde{R}$ is not Cohen-Macaulay? The answer is yes, as we see next.

\section{Non-COHEn-Macaulay CANONical COVERS OF RINGS WITH RATIONAL SINGULARITIES}

We show that the canonical cover of a ring with rational singularities need not be Cohen-Macaulay:

Theorem 6.1. For all $d \geq 3$, there exists a graded $\mathbb{Q}$-Gorenstein ring $R$ of dimension $d$, with an isolated rational singularity, whose canonical cover $\widetilde{R}$ has depth 2 . In particular, $\widetilde{R}$ is not a Cohen-Macaulay ring.

Proof. Let $K$ be an algebraically closed field of characteristic 0 . The ring $R$ in the statement of the theorem will be constructed as a Segre product $A \# B$. For the ring $A$, as in Example 4.4 take the subring of

$$
\widetilde{A}=K[X, Y, Z] /\left(X^{3}-Y Z(Y+Z)\right)
$$

generated by the elements $y^{3}, y^{2} z, y z^{2}, z^{3}$ and $x$. 
Case $d=3: \quad$ On $\mathbb{P}^{1}=\operatorname{Proj} K\left[v_{0}, w_{0}\right]$, take the $\mathbb{Q}$-divisor

$$
E=\frac{1}{2} V\left(v_{0}\right)+\frac{1}{2} V\left(w_{0}\right)+\frac{1}{2} V\left(v_{0}-w_{0}\right)+\frac{1}{2} V\left(v_{0}+w_{0}\right)
$$

and set $B=\bigoplus_{n \geq 0} H^{0}\left(\mathbb{P}^{1}, \mathcal{O}_{\mathbb{P}^{1}}(n E)\right) u^{n}$. The generators of $B$ are

$$
\begin{aligned}
& \frac{v_{0}^{4} u^{2}}{v_{0} w_{0}\left(v_{0}^{2}-w_{0}^{2}\right)}, \frac{v_{0}^{3} w_{0} u^{2}}{v_{0} w_{0}\left(v_{0}^{2}-w_{0}^{2}\right)}, \frac{v_{0}^{2} w_{0}^{2} u^{2}}{v_{0} w_{0}\left(v_{0}^{2}-w_{0}^{2}\right)}, \frac{v_{0} w_{0}^{3} u^{2}}{v_{0} w_{0}\left(v_{0}^{2}-w_{0}^{2}\right)}, \\
& \frac{w_{0}^{4} u^{2}}{v_{0} w_{0}\left(v_{0}^{2}-w_{0}\right)}, \quad \text { and } u,
\end{aligned}
$$

and we identify $B$ with the subring of the hypersurface

$$
K[U, V, W] /\left(U^{2}-V W\left(V^{2}-W^{2}\right)\right)
$$

generated by the elements $v^{4}, v^{3} w, v^{2} w^{2}, v w^{3}, w^{4}$, which are assigned weight 2 , and $u$, which has weight 1 . A graded canonical module for $B$ is

$$
\omega_{B}=\frac{1}{u}\left(v^{4}, v^{3} w, v^{2} w^{2}\right) B
$$

This has order 2 as a divisor class group element, and $\omega_{B}^{(2)}=\frac{v^{4}}{u^{2}} B$. The canonical cover $\widetilde{B}$ is isomorphic to the subring of the hypersurface generated by $v^{2}, v w, w^{2}$ and $u$.

Case $d \geq 4: \quad$ In the situation of Example 3.4 take $r=2, m=d-1$, and $n=2 m$, i.e., the $\mathbb{Q}$-divisor

$$
E=\frac{1}{2} V\left(X_{1}^{2 m}+\cdots+X_{m}^{2 m}\right) \quad \text { on } \quad \mathbb{P}^{m-1}=\operatorname{Proj} K\left[X_{1}, \ldots, X_{m}\right],
$$

and the generalized section ring $B=\bigoplus_{n \geq 0} H^{0}\left(\mathbb{P}^{m-1}, \mathcal{O}_{\mathbb{P}} m-1(n E)\right) Z^{n}$. Note that $B$ is not of dense F-regular type, since $\operatorname{deg}\left(K_{\mathbb{P}^{m-1}}+E^{\prime}\right)=0$. We identify $B$ with the subring of the hypersurface

$$
K\left[Z, X_{1}, \ldots, X_{m}\right] /\left(Z^{2}-\left(X_{1}^{2 m}+\cdots+X_{m}^{2 m}\right)\right)
$$

generated by $z$ and monomials of degree $2 m$ in $x_{1}, \ldots, x_{m}$. It is easily checked (using the corresponding $\mathbb{Q}$-divisors) that as a graded canonical module for $B$, we may take the fractional ideal

$$
\omega_{B}=\frac{1}{z}\left(x_{1}^{m} \mu_{m}: \mu_{m} \text { runs through degree } m \text { monomials in } x_{1}, \ldots, x_{m}\right) B .
$$

Its second symbolic power is $\omega_{B}^{(2)}=\left(x_{1}^{2 m} / z^{2}\right) B$.

Note that the order of $\omega_{A}$ in $\mathrm{Cl}(A)$ is 3 , and (in either case) that $\omega_{B}$ has order 2 as an element of $\mathrm{Cl}(B)$. Furthermore, $a(\widetilde{A})=a(\widetilde{B})=0$. So by Proposition 4.5, the Segre product $R=A \# B$ is $\mathbb{Q}$-Gorenstein, and its canonical cover $\widetilde{R}$ is isomorphic to $\widetilde{A} \# \widetilde{B}$. By Proposition 2.3, $A$ and $B$ are rings with rational singularities over an algebraically closed field $K$, and it follows that $A \otimes_{K} B$ has rational singularities. By Boutot's theorem, $[\mathrm{Bo}]$, its direct summand $R=A \# B$ has rational singularities as well. The verification that $R$ has an isolated singularity at $m_{R}$ is rather routine and is left to the reader. 
It remains to check that the canonical cover of $R$, i.e., $\widetilde{R} \cong \widetilde{A} \# \widetilde{B}$, has depth 2 . Since $\operatorname{dim} \widetilde{A}=2$, by the Künneth formula for local cohomology we have

$$
H_{m_{\widetilde{R}}}^{2}(\widetilde{R}) \cong\left(\widetilde{A} \# H_{m_{\widetilde{B}}}^{2}(\widetilde{B})\right) \oplus\left(H_{m_{\widetilde{A}}}^{2}(\widetilde{A}) \# \widetilde{B}\right)
$$

which is nonzero since $a(\widetilde{A})=0$.

\section{REFERENCES}

[Bo] J.-F. Boutot, Singularités rationnelles et quotients par les groupes réductifs, Invent. Math. 88 (1987), 65-68. MR 88a:14005

[Ch] W.-L. Chow, On unmixedness theorem, Amer. J. Math. 86 (1964), 799-822. MR 30:2031

[De] M. Demazure, Anneaux gradués normaux, in: Lẽ Dûng Tráng (ed.) Introduction à la théorie des singularités II, Hermann, Paris, (1988), 35-68. MR 91k:14004

[GoW] S. Goto and K.-i. Watanabe, On graded rings I, J. Math. Soc. Japan 30 (1978), 179-213. MR 81m:13021

[GrW] P. Griffith and D. Weston, Restrictions of torsion divisor classes to hypersurfaces, J. Algebra 167 (1994), 473-487. MR 95c:13008

[Gr] P. Griffith, Induced formal deformations and the Cohen-Macaulay property, Trans. Amer. Math. Soc. 353 (2001), 77-93. MR 2001b:13020

[Ha] N. Hara, A characterisation of rational singularities in terms of injectivity of Frobenius maps, Amer. J. Math. 120 (1998), 981-996. MR 99h:13005

[HW] N. Hara and K.-i. Watanabe, F-regular and F-pure rings vs. log terminal and log canonical singularities, J. Algebraic Geom. 11 (2002), 363-392.

[HH1] M. Hochster and C. Huneke, Tight closure and strong F-regularity, Mem. Soc. Math. France 38 (1989), 119-133. MR 91i:13025

[HH2] M. Hochster and C. Huneke, Tight closure, invariant theory, and the Briançon-Skoda theorem, J. Amer. Math. Soc. 3 (1990), 31-116. MR 91g:13010

[HH3] M. Hochster and C. Huneke, F-regularity, test elements, and smooth base change, Trans. Amer. Math. Soc. 346 (1994), 1-62. MR 95d:13007

[HH4] M. Hochster and C. Huneke, Tight closure of parameter ideals and splitting in module-finite extensions, J. Algebraic Geom. 3 (1994), 599-670. MR 95k:13002

[HH5] M. Hochster and C. Huneke, Tight closure in equal characteristic zero, in preparation.

[HR] M. Hochster and J. Roberts, Rings of invariants of reductive groups acting on regular rings are Cohen-Macaulay, Adv. in Math. 13 (1974), 115-175. MR 50:311

[Ka] Y. Kawamata, The cone of curves of algebraic varieties, Ann. of Math. (2) 119 (1984), 603-633. MR 86c:14013b

[Li] J. Lipman, Rational singularities, with applications to algebraic surfaces and unique factorization, Inst. Hautes Études Sci. Publ. Math. 36 (1969), 195-279. MR 43:1986

[LS] G. Lyubeznik and K. E. Smith, Strong and weak F-regularity are equivalent for graded rings, Amer. J. Math. 121 (1999), 1279-1290. MR 2000m:13006

[Ma] H. Matsumura, Commutative ring theory, Cambridge Studies in Advanced Mathematics 8, Cambridge University Press, Cambridge-New York, 1986. MR 88h:13001

[Si1] A. K. Singh, $\mathbb{Q}$-Gorenstein splinter rings of characteristic $p$ are F-regular, Mathematical Proceedings of the Cambridge Philosophical Society 127 (1999), 201-205. MR 2000j:13006

[Si2] A. K. Singh, Multi-symbolic Rees algebras and strong F-regularity, Math. Z. 235 (2000), 335-344. MR 2001j:13006

[Sm1] K. E. Smith, F-rational rings have rational singularities, Amer. J. Math. 119 (1997), 159-180. MR 97k:13004

[Sm2] K. E. Smith, Vanishing theorems, singularities, and effective bounds in algebraic geometry via prime characteristic local algebra, in: J. Kollár, R. Lazarsfeld and David R. Morrison (eds.) Proc. Sympos. Pure Math. 62 (1997), 289-325. MR 99a:14026

[TW] M. Tomari and K.-i. Watanabe, Normal $\mathbf{Z}_{r}$-graded rings and normal cyclic covers, Manuscripta Math. 76 (1992), 325-340. MR 93j:13002

[Wa1] K.-i. Watanabe, Some remarks concerning Demazure's construction of normal graded rings, Nagoya Math. J. 83 (1981), 203-211. MR 83g:13016 
[Wa2] K.-i. Watanabe, Rational singularities with $k^{*}$-action, in: Commutative Algebra (Trento, 1981), Lecture Notes in Pure and Appl. Math. 84, Marcel Dekker, New York, (1983), 339-351. MR 84e:14005

[Wa3] K.-i. Watanabe, F-regular and F-pure normal graded rings, J. Pure Appl. Algebra 71 (1991), 341-350. MR 92g:13003

[Wa4] K.-i. Watanabe, Infinite cyclic covers of strongly F-regular rings, Contemp. Math. 159 (1994), 423-432. MR 95c:13030

Department of Mathematics, University of Utah, 155 S. 1400 E., Salt Lake City, UTAн 84112-0090

Current address: Mathematical Sciences Research Institute, 1000 Centennial Drive, \#5070, Berkeley, California 94720-5070

E-mail address: asingh@msri.org 